\title{
A Sequence Selection Bound for the Capacity of the Nonlinear Fiber Channel
}

\author{
Stella Civelli ${ }^{(1,2)}$, Enrico Forestieri(1,2), Alexey Lotsmanov ${ }^{(3)}$, Dmitry Razdoburdin( ${ }^{(3,4)}$, Marco Secondini ${ }^{(1,2)}$
}

(1) Tecip Institute, Scuola Superiore Sant'anna stella.civelli@santannapisa.it

(2) PNTLab, Consorzio nazionale interuniversitario per le telecomunicazioni (CNIT)

(3) Moscow Research Center, Huawei Technologies Co., Ltd., Moscow, Russia

(4) Sternberg Astronomical Institute, Moscow M.V. Lomonosov State University, Moscow, Russia

\begin{abstract}
A novel technique to optimize the input distribution and compute a lower bound for the capacity of the nonlinear optical fiber channel is proposed. The technique improves previous bounds obtained with the additive white Gaussian noise decoding metric.
\end{abstract}

\section{Introduction}

The capacity of the fiber channel in the nonlinear regime is not known [1- [1] , but only upper-limited by the linear capacity ${ }^{4}$ and lower-limited by numerous bounds [5]-112]. Most of the bounds are obtained by computing an achievable information rate (AIR) with an optimized decoding metric, while considering a simple fixed input distribution-typically, i.i.d. samples with Gaussian distribution [5], [6], [8] - [12] or multiple rings with uniform phase ${ }^{7}$.

In this work, we propose a novel sequence selection technique to optimize the input distribution and compute an improved lower bound for the capacity of the nonlinear fiber channel. The technique uses a rejection sampling method to select only the sequences of symbols that generate less nonlinear interference. The AIR achievable when encoding information on the selected sequences is then lower bounded by removing the rate loss caused by the selection procedure.

\section{Sequence selection}

The sequence selection procedure-sketched in Fig. 1-is a sort of rejection sampling method, in which a random sequence of $N$ symbols $\mathrm{x}$ is drawn from a given unbiased source with probability distribution $P(\mathbf{x})$, accepted if it meets a certain condition, or rejected otherwise. The combination of the unbiased source and the rejection method forms the biased source, which generates the symbols at the channel input. The unbiased source can be arbitrarily selected, i.e., the $N$ symbols can be drawn from a continuous (e.g., Gaussian) or discrete (e.g., quadrature amplitude modulation (QAM)) constellation, and can be independent or correlated (e.g., obtained as the output of a finiteblock-length distribution matcher that implements

978-1-6654-3868-1/21/\$31.00 @2021 IEEE

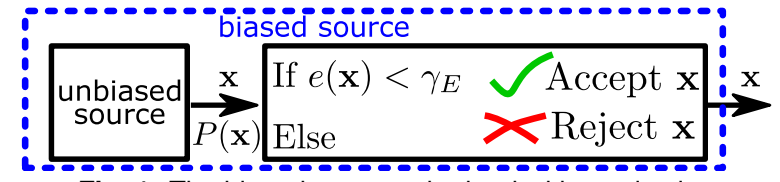

Fig. 1: The biased source, obtained with a selection procedure from an unbiased source.

probabilistic shaping (PS). The acceptance criterion is defined by selecting a proper metric $e(\cdot)$ which measures the amount of nonlinear interference generated by a sequence-and a threshold $\gamma_{E}$, so that a sequence is accepted only if the metric $e(\mathbf{x})$ is below the threshold, i.e., if it generates a small amount of nonlinear interference. The probability of the sequences generated by the biased source is

$$
P_{b}(\mathbf{x})= \begin{cases}P(\mathbf{x}) / \eta & e(\mathbf{x})<\gamma_{E} \\ 0 & e(\mathbf{x}) \geq \gamma_{E}\end{cases}
$$

where $\eta=\operatorname{Pr}\left\{e(\mathbf{x})<\gamma_{E}\right\}$ is the acceptance probability, which can be approximated as $\eta \approx N_{s} / N_{t}$ where $N_{s}$ is the number of selected sequences and $N_{t}$ is the number of tested sequences.

The selection metric $e(\cdot)$ can be defined and computed in different ways. In this work, we consider only intrachannel nonlinearity, estimated by using the split-step Fourier method (SSFM). The metric is defined as $e(\mathbf{x})=\|\mathbf{x}-\mathbf{y}\|$, where $\mathbf{x}$ is the transmitted sequence and $\mathrm{y}$ is the corresponding received sequence after a single-channel noiseless propagation. The sequences from the biased source are obtained by the following steps:

1. Draw $N_{t}$ test sequences $\left\{\mathbf{x}_{k}\right\}$, with $k=$ $1, \ldots, N_{t}$, from the unbiased source.

2. Form the sequence $\mathbf{s}=\left(\mathbf{x}_{1}, \ldots, \mathbf{x}_{N_{t}}\right)$ of length $N_{t} N$, obtained by concatenating all the test sequences.

3. Run a single-channel noiseless simulation with input $\mathbf{s}$, including all the steps that 
will be included in the system except for digital backpropagation (DBP)-i.e., modulation, SSFM propagation, dispersion compensation, matched filtering and sampling, mean phase rotation compensation-to obtain the corresponding received sequence $\mathbf{r}=\left(\mathbf{y}_{1}, \ldots, \mathbf{y}_{N_{t}}\right)$.

4. Accept only the $N_{s}$ sequences with $e\left(\mathbf{x}_{k}\right)=$ $\left\|\mathbf{x}_{k}-\mathbf{y}_{k}\right\|<\gamma_{E}$.

As far as it concerns the system performance, we expect it to improve as $\gamma_{E}$ becomes smalleri.e., as the maximum amount of nonlinear interference that can be generated by each sequence diminishes - at least in the same scenario considered for sequence optimization (single-channel, dispersion compensation only). However, by reducing $\gamma_{E}$ also $\eta$ decreases, meaning that less sequences are available to encode information. This rate loss is accounted for in the computation of the AIR. When the transmitted symbols are drawn from a Gaussian constellation, we consider the AIR with symbol-wise decoding, $\mathrm{AIR}_{\mathrm{s}}$. When sequence selection is not applied, $\mathrm{AIR}_{\mathrm{s}}$ is evaluated as in 13 , assuming a mismatched decoding metric optimized for the additive white Gaussian noise (AWGN) channel. Conversely, when sequence selection is applied, the following lower bound holds

$$
\mathrm{AIR}_{\mathrm{s}} \geq \operatorname{AIR}_{\mathrm{s}}^{(\mathrm{u})}+\frac{\log _{2} \eta}{2 N} \text { in bits/symbol/pol }
$$

where $\operatorname{AIR}_{\mathrm{s}}^{(\mathrm{u})}$ is evaluated assuming that the received sequence $\mathbf{x}$ has unbiased probability $P(\mathbf{x})$, i.e., transmitting the sequences obtained from the biased source but computing the AIR with the same expression as in the case without sequence selection. The inequality in (2) is obtained using $P_{b}(\mathbf{x}) \leq \eta P(\mathbf{x})$, which follows from (1) and implies a loss of at most $\log _{2}(1 / \eta)$ bits on a sequence of $N$ 2-polarization symbols.

On the other hand, when the transmitted symbols are drawn from a QAM constellation, we consider the AIR with bit-wise decoding, $\mathrm{AIR}_{\mathrm{b}}$, still with the same mismatched AWGN decoding metric. When sequence selection is not applied, $\mathrm{AIR}_{\mathrm{b}}$ is evaluated as in 144, 15. When sequence selection is applied, a lower bound analogous to (2) holds.

\section{System setup and results}

The system setup is the same considered in 13 . A dual polarization WDM signal made of $5 R_{s}=$ $50 \mathrm{GBd}$ channels, with $50 \mathrm{GHz}$ spacing, sinc pulses, and Gaussian or QAM symbols with PS, is launched into a $1000 \mathrm{~km}$ link. The link is

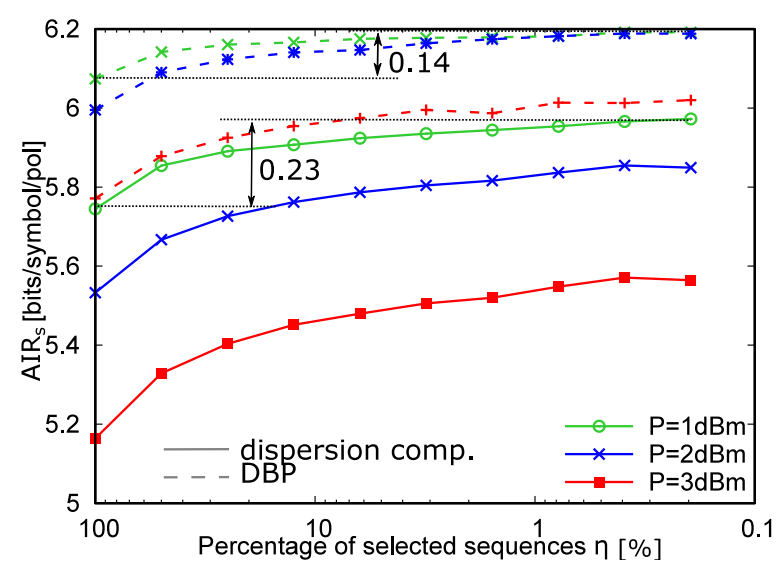

Fig. 2: $A_{I R}$ vs selection rate for the EDFA link and i.i.d. Gaussian symbols as unbiased source.

made of $10 \times 100 \mathrm{~km}$ spans of single mode fiber $\left(D=17 \mathrm{ps} / \mathrm{nm} / \mathrm{km}, \gamma=1.3 \mathrm{~W}^{-1} / \mathrm{km}\right.$, and $\alpha_{\mathrm{dB}}=$ $0.2 \mathrm{~dB} / \mathrm{km}$ ), after each span an erbium-doped fiber amplifier (EDFA) with spontaneous emission coefficient equal to 1 compensates for loss. The ideal Raman amplification (IDRA) case is also considered. At the RX, the central channel is demultiplexed, DBP or dispersion compensation is applied, followed by matched filter and sampling at symbol time $1 / R_{s}$. After a mean phase rotation removal, the lower bound to the $\mathrm{AIR}_{\mathrm{s}}$ or $\mathrm{AIR}_{\mathrm{b}}$ is evaluated. The PS is implemented through the probabilistic amplitude shaping (PAS) approach ${ }^{16}$, using either i.i.d. symbols drawn from a MaxwellBoltzmann (MB) distribution-optimal in the linear regime-or the enumerative sphere shaping (ESS) [17] with optimized block length equal to 256 . The length of the sequences is $N=2562$-pol QAM symbols. The number of tested sequences is $N_{t}=2^{16}$. The selection procedure to determine which sequences are accepted (used in simulations to compute the AIR) or rejected is performed in a noiseless single-channel scenario at a launch power corresponding to the optimal power for the case without sequence selection.

Fig. 2 shows the lower bound (2) as a function of the selection rate $\eta$ for the EDFA link and different launch powers, both without DBP (solid lines) and with ideal single-channel DBP (dashed lines). The optimal power is $P=1 \mathrm{dBm}$ at $\eta=100 \%$. The figure shows that the performance improves as $\eta$ decreases, though it seems to approach a maximum near the smallest value of $\eta$ considered in the figure (which is limited by the number of tested sequences). This happens because (i) the sequences have been optimized for the single channel scenario without DBP, and not for the consider scenario, making the sequences not optimal, and (ii) the loss due to sequence selection-the sec- 


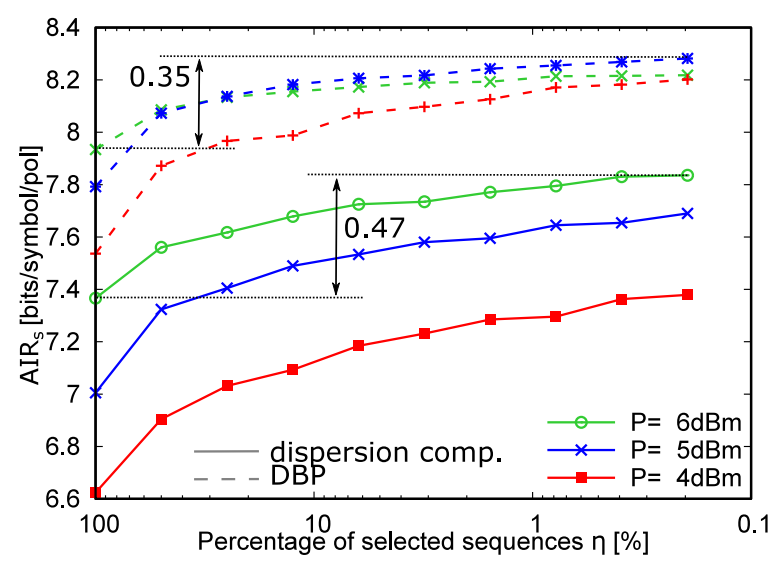

Fig. 3: $\mathrm{AIR}_{\mathrm{S}}$ vs selection rate for the IDRA link and i.i.d. Gaussian symbols as unbiased source.

ond term in (2)-increases when $\eta$ decreases, so that eventually all the curve must decrease again and vanish when $\eta \rightarrow 0$. Overall, the maximum gain in the case without DBP is $0.23 \mathrm{bits} / \mathrm{symbol} / \mathrm{pol}$ obtained with $\eta=0.19 \%$, and $0.14 \mathrm{bits} / \mathrm{symbol} / \mathrm{pol}$ obtained with $\eta=0.39 \%$ for the case with DBP. The gain obtained with DBP-though smaller-is particularly interesting since the sequences have been selected in the single channel scenario without DBP, that is, to minimize the intrachannel nonlinearity, which is instead fully compensated for by DBP in this case. This means that the same sequences that are "good" for intrachannel nonlinearity are good also for mitigating interchannel nonlinearities. However, we expect to achieve higher gains by employing a selection metric that measures also interchannel nonlinearity.

Fig. 3 shows the results for the IDRA link. The qualitative behaviour is the same as in the EDFA link of Fig. 2, but with larger gains: the maximum gains are $0.47 \mathrm{bits} / \mathrm{symbol} / \mathrm{pol}$ and $0.35 \mathrm{bits} / \mathrm{symbol} / \mathrm{pol}$ without and with DBP, respectively. The reason why the gains are higher in the IDRA link than in the EDFA link is under investigation, but we note that the same behaviour is observed in the case studied in $[13]$, where it can be explained by the higher coherence (in time and frequency) of the cross-phase modulation term in the IDA link. In $\frac{13}{13}$, however, the AIR gains are obtained by optimizing the decoding metric rather than the input distribution. Moreover, we expect that by combining the sequence selection approach proposed here for the optimization of the input distribution with the optimized decoding metric employed in [12], 13], we might further improve the AIR (and capacity bounds).

Finally, we test the proposed approach for a discrete constellation. Fig. 4 shows the performance of sequence selection when a PAS-256 QAM con-

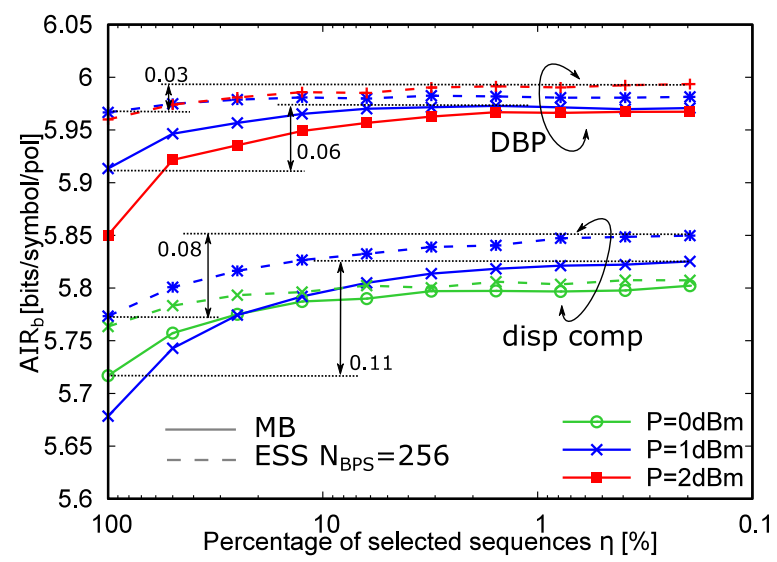

Fig. 4: $\mathrm{AIR}_{\mathrm{b}}$ vs selection rate for the EDFA link and PAS-256 QAM symbols with i.i.d. MB distribution (solid lines) or drawn by ESS with optimized block length (dashed lines) as unbiased source.

stellation with rate $R=6.4 \mathrm{bits} / \mathrm{symbol} / \mathrm{pol}$ is used, with or without DBP. When i.i.d. symbols with the MB distribution are considered as a starting point $(\eta=100 \%)$ for the unbiased distribution (solid lines), the performance improves by $0.11 \mathrm{bits} / \mathrm{symbol} / \mathrm{pol}$ and $0.06 \mathrm{bits} / \mathrm{symbol} / \mathrm{pol}$, without and with DBP, respectively. Interestingly, the gain provided by sequence selection in the case without DBP is 0.05 bits/symbol/pol larger than the gain provided by PAS with optimized block length ${ }^{18}-20$. . When DBP is used, the two techniques-sequence selection with MB symbols and optimized ESS without sequence selectionprovide the same gain. Finally, the highest AIR is obtained when considering ESS with optimized block length [19] as a starting point $(\eta=100 \%)$ for the unbiased distribution (dashed lines). In this case, sequence selection yields a gain of $0.08 \mathrm{bits} / \mathrm{symbol} / \mathrm{pol}$ and $0.03 \mathrm{bits} / \mathrm{symbol} / \mathrm{pol}$ without and with DBP, respectively.

\section{Conclusions}

We have proposed a novel sequence selection technique to lower-bound the capacity of the nonlinear optical fiber channel. Using a simple numerical optimization, the proposed technique improves the AIR obtained with an AWGN decoding metric, with significant gains over both EDFA and IDRA links, with both continuous and discrete constellations. The use of a more accurate selection metric (e.g., accounting also for interchannel nonlinearity) and the combination with an improved decoding metric $\left.{ }^{12]}, 13\right]$ are expected to further increase the lower bounds provided in this work.

\section{Acknowledgement}

This work was funded in part by Huawei. 


\section{References}

[1] M. Secondini and E. Forestieri, "Scope and limitations of the nonlinear Shannon limit," J. Lightw. Technol., vol. 35, no. 4, pp. 893-902, Feb. 2017.

[2] E. Agrell, "Capacity bounds in optical communications," in Proc. Eur. Conf. Opt. Commun. (ECOC), Sep. 2017.

[3] M. Secondini, "Chapter 20 - information capacity of optical channels," in Optical Fiber Telecommunications VII, A. E. Willner, Ed., Academic Press, 2020, pp. 867-920, ISBN: 978-0-12-816502-7. DOI: https://doi.org/10. 1016/B978-0-12-816502-7.00023-3 [Online]. Available: https : / / www . sciencedirect . com / science / article/pii/B9780128165027000233

[4] G. Kramer, M. I. Yousefi, and F. R. Kschischang, "Upper bound on the capacity of a cascade of nonlinear and noisy channels," in IEEE Information Theory Workshop (ITW), Apr. 2015.

[5] A. Splett, C. Kurtzke, and K. Petermann, "Ultimate transmission capacity of amplified optical fiber communication systems taking into account fiber nonlinearities," in Proc. Eur. Conf. Opt. Commun. (ECOC), vol. 2, 1993, pp. 41-44.

[6] P. P. Mitra and J. B. Stark, "Nonlinear limits to the information capacity of optical fiber communications," Nature, vol. 411, no. 6841, pp. 1027-1030, Jun. 2001.

[7] R.-J. Essiambre, G. Kramer, P. J. Winzer, G. J. Foschini, and B. Goebel, "Capacity limits of optical fiber networks," J. Lightwave Technol., vol. 28, no. 4, pp. 662-701, Feb. 2010.

[8] A. Mecozzi and R.-J. Essiambre, "Nonlinear Shannon limit in pseudolinear coherent systems," J. Lightw. Technol., vol. 30, no. 12, pp. 2011-2024, Jun. 2012.

[9] R. Dar, M. Shtaif, and M. Feder, "New bounds on the capacity of the nonlinear fiber-optic channel," vol. 39, no. 2, pp. 398-401, Jan. 2014.

[10] M. Secondini, E. Agrell, E. Forestieri, and D. Marsella, "Fiber nonlinearity mitigation in WDM systems: Strategies and achievable rates," in Proc. Eur. Conf. Opt. Commun. (ECOC), Sep. 2017.

[11] F. J. García-Gómez and G. Kramer, "Mismatched models to lower bound the capacity of optical fiber channels," Journal of Lightwave Technology, vol. 38, no. 24, pp. 6779-6787, 2020.

[12] _ "Mismatched models to lower bound the capacity of dual-polarization optical fiber channels," Journal of Lightwave Technology, 2021.

[13] M. Secondini, E. Agrell, E. Forestieri, D. Marsella, and M. R. Camara, "Nonlinearity mitigation in WDM systems: Models, strategies, and achievable rates," Journal of Lightwave Technology, vol. 37, no. 10, pp. 2270-2283, 2019.

[14] T. Fehenberger, A. Alvarado, G. Böcherer, and N. Hanik, "On probabilistic shaping of quadrature amplitude modulation for the nonlinear fiber channel," Journal of Lightwave Technology, vol. 34, no. 21, pp. 5063-5073, 2016.

[15] A. Alvarado, T. Fehenberger, B. Chen, and F. M. Willems, "Achievable information rates for fiber optics: Applications and computations," Journal of Lightwave Technology, vol. 36, no. 2, pp. 424-439, 2018.
[16] G. Böcherer, F. Steiner, and P. Schulte, "Bandwidth efficient and rate-matched low-density parity-check coded modulation," IEEE Transactions on Communications, vol. 63, no. 12, pp. 4651-4665, 2015.

[17] Y. Gultekin, W. van Houtum, and F. Willems, "On constellation shaping for short block lengths," in 2018 Symposium on Information Theory and Signal Processing in the Benelux (SITB 2018), University of Twente, 2018, pp. 86-96.

[18] O. Geller, R. Dar, M. Feder, and M. Shtaif, "A shaping algorithm for mitigating inter-channel nonlinear phasenoise in nonlinear fiber systems," Journal of Lightwave Technology, vol. 34, no. 16, pp. 3884-3889, 2016.

[19] S. Civelli, E. Forestieri, and M. Secondini, "Interplay of probabilistic shaping and carrier phase recovery for nonlinearity mitigation," in 2020 European Conference on Optical Communication (ECOC), IEEE, 2020, pp. 13.

[20] T. Fehenberger, H. Griesser, and J.-P. Elbers, "Mitigating fiber nonlinearities by short-length probabilistic shaping," in Optical Fiber Communication Conference, Optical Society of America, 2020, Th11-2. 